\title{
Revised Recommendations of the Consortium of MS Centers Task Force for a Standardized MRI Protocol and Clinical Guidelines for the Diagnosis and Follow-Up of Multiple Sclerosis
}

\author{
(D) A. Traboulsee, J.H. Simon, L. Stone, E. Fisher, D.E. Jones, A. Malhotra, S.D. Newsome, J. Oh, DD.S. Reich, N. Richert, K. Rammohan,
} O. Khan, E.-W. Radue, C. Ford, J. Halper, and (DD. Li

\begin{abstract}
SUMMARY: An international group of neurologists and radiologists developed revised guidelines for standardized brain and spinal cord MR imaging for the diagnosis and follow-up of MS. A brain MR imaging with gadolinium is recommended for the diagnosis of MS. A spinal cord MR imaging is recommended if the brain MR imaging is nondiagnostic or if the presenting symptoms are at the level of the spinal cord. A follow-up brain MR imaging with gadolinium is recommended to demonstrate dissemination in time and ongoing clinically silent disease activity while on treatment, to evaluate unexpected clinical worsening, to re-assess the original diagnosis, and as a new baseline before starting or modifying therapy. A routine brain MR imaging should be considered every 6 months to 2 years for all patients with relapsing MS. The brain MR imaging protocol includes 3D T1-weighted, 3D T2-FLAIR, 3D T2-weighted, post-single-dose gadolinium-enhanced T7-weighted sequences, and a DWI sequence. The progressive multifocal leukoencephalopathy surveillance protocol includes FLAIR and DWI sequences only. The spinal cord MR imaging protocol includes sagittal Tl-weighted and proton attenuation, STIR or phase-sensitive inversion recovery, axial T2- or T2*-weighted imaging through suspicious lesions, and, in some cases, postcontrast gadolinium-enhanced T7-weighted imaging. The clinical question being addressed should be provided in the requisition for the MR imaging. The radiology report should be descriptive, with results referenced to previous studies. MR imaging studies should be permanently retained and available. The current revision incorporates new clinical information and imaging techniques that have become more available.
\end{abstract}

ABBREVIATIONS: $\mathrm{CIS}=$ clinically isolated syndrome; $\mathrm{CMSC}=$ Consortium of MS Centers; $\mathrm{PML}=$ progressive multifocal leukoencephalopathy

$\mathrm{M}$ $\mathrm{R}$ imaging of the brain and spinal cord is sensitive for detecting white matter lesions typical of MS. The current diagnostic criteria for $\mathrm{MS}^{1}$ include specific MR imaging features

From the Department of Medicine (Neurology) (A.T.), University of British Columbia, Vancouver, Canada; Portland VA Research Foundation and Oregon Health and Sciences University (J.H.S.), Portland, Oregon; Mellen Center for MS Treatment and Research (L.S.), Cleveland Clinic, Cleveland, Ohio; Department of Biomedical Engineering, Cleveland Clinic (E.F.). Cleveland, Ohio; Department of Neurology, University of Virginia (D.E.J.), Charlottesville, Virginia; Department of Radiology and Biomedical Imaging, Yale University (A.M.), New Haven, Connecticut; Department of Neurology (S.D.N.), Johns Hopkins School of Medicine, Baltimore, Maryland; St. Michael's Hospital (J.O.), University of Toronto, Toronto, Ontario, Canada; Translational Neuroradiology Unit (D.S.R.), National Institute of Neurological Disorders and Stroke, National Institutes of Health, Bethesda, Maryland; Biogen Idec (N.R.), Cambridge, Massachusetts; University of Miami Multiple Sclerosis Center (K.R.), Miami, Florida; Department of Neurology (O.K.), Wayne State University School of Medicine, Detroit, Michigan; Department of Radiology (E.-W.R.), University Hospital, Basel, Switzerland; University of New Mexico Health Science Center (C.F.), Albuquerque, New Mexico; Consortium of Multiple Sclerosis Centers (J.H.), Hackensack, New Jersey; and Departments of Radiology (D.L.), University of British Columbia, Vancouver, British Columbia Canada.

Organizational support and funding for the meetings were provided by the Consortium of Multiple Sclerosis Centers (www.mscare.org).

Please address correspondence to Anthony Traboulsee, MD, Department of Medicine (Neurology), University of British Columbia, UBC Hospital, 2211 Wesbrook Mall, Room s199, Vancouver, BC, Canada V6T 2B5; e-mail: t.traboulsee@ubc.ca

- Indicates open access to non-subscribers at www.ajnr.org

http://dx.doi.org/10.3174/ajnr.A4539
(Table 1) to provide evidence for dissemination in space and/or time, allowing an earlier diagnosis of MS after a single clinical syndrome consistent with demyelination (clinically isolated syndrome [CIS]). The newer criteria have good sensitivity and specificity $^{2}$ compared with the prior clinical criteria. ${ }^{3}$ However, white matter lesions are common in the general population with increasing age, and the MR imaging criteria should be used with caution in patients with atypical symptoms for MS or the onset of symptoms in patients older than 40 years of age. This recommendation is particularly important in the presence of factors known to cause T2 hyperintensities, including hypertension, smoking, diabetes, high cholesterol, and migraines.

MR imaging is also increasingly used to follow patients with a diagnosis of definite MS to determine progression of clinically silent disease activity and to monitor response to therapy. Gadolinium (contrast)-enhancing lesions and/or changes in T2 (hyperintense) lesions are accepted MR imaging biomarkers of new inflammation. New MR imaging activity occurs more frequently than new clinical symptoms (relapses). ${ }^{4}$ The ability to monitor patients with MS with MR imaging is hampered by inconsistent protocols and image quality.

Recommendations for a standardized MR imaging protocol and clinical guidelines in MS were previously published. ${ }^{5}$ These 
developed out of a series of meetings sponsored by the Consortium of MS Centers (CMSC), including radiologists and neurologists from academic and community-based MS practices and representatives of the American Academy of Neurology, the Radiological Society of North America, and the American Society of Neuroradiology. The goal of this article is to update the MR imaging protocol and clinical guidelines on the basis of advances in imaging technology and new clinical evidence of the role of MR imaging in the diagnosis and monitoring of MS.

\section{Methods}

Neurologists, radiologists, and imaging scientists with an expertise in MS from North America and Europe, representatives of the American Academy of Neurology, the Radiological Society of North America, the American Society of Neuroradiology, and, more recently, the National Institutes of Health and the North American Imaging in Multiple Sclerosis Cooperative updated the guidelines on the basis of new data, survey results, and expert opinion. Four imaging protocols, routine brain, progressive multifocal leukoencephalopathy (PML) surveillance, spinal cord, and orbits, were developed. Clinical guidelines on the recommended frequency of imaging in diagnosing and monitoring MS were updated.

Protocol 1: Brain MR Imaging. The brain MR imaging protocol (Table 2 and Fig 1) provides the minimum required sequence to aid

Table 1: 2010 Revised McDonald diagnostic criteria for MS $^{\mathbf{a}}$ Minimum MRI Features for DIS ( 2 of 4 Criteria Required)

\section{Infratentorial lesion}

1 Juxtacortical lesion (touching the cortex)

1 Periventricular lesion (touching the ventricles)

1 Spinal cord lesion

Note:-DIS indicates dissemination in space; DIT, dissemination in time.

${ }^{a}$ MS diagnosis requires clinical and/or MRI evidence for CNS demyelination occurring in multiple locations (DIS) and with multiple events (DIT). The MRI criteria may support the clinical diagnosis of patients with MS with typical symptoms of CNS demyelination after the exclusion of alternative diagnoses. The DIT criterion can be met on MRI with an asymptomatic contrast-enhancing lesion on TTWI sequences (first or follow-up MRI) or newly active T2WI lesions on follow-up MRI. Lesions should be at least 3-mm in diameter and asymptomatic. in the diagnosis and monitoring of MS that can be performed on a variety of clinical scanners and includes 3D T1-weighted, 3D T2FLAIR, 3D T2-weighted, and post-single-dose gadolinium-enhanced T1-weighted imaging, all with a nongapped section thickness of $\leq 3 \mathrm{~mm}$, and a DWI sequence ( $\leq 5-\mathrm{mm}$ section thickness). Additional sequences for non-MS pathology can be added, depending on the individual needs of the patient and local preferences.

Scans should be of good quality with adequate SNR and spatial resolution (in-section pixel resolution of $\leq 1 \times 1 \mathrm{~mm}$ ). Reconstruction (interpolation) is recommended at $0.5 \mathrm{~mm}$. This recommendation may be limiting for some older scanners, particularly those operating at lower field strengths. One needs to be aware of the higher lesion-detection rates at 3T compared with $1.5 \mathrm{~T}$. $^{6}$ Lower field (ie, "open magnet") should only be used in extenuating circumstances.

Coverage should include the whole brain. Orientation of the axial sequences (acquisition of 2D sequences or reformatting of 3D sequences) should be along the subcallosal line (Fig 1) because consistent repositioning is essential for detecting changes across time.

Most scanners are capable of $3 \mathrm{D}$ acquisitions with $\leq 1.2-\mathrm{mm}$

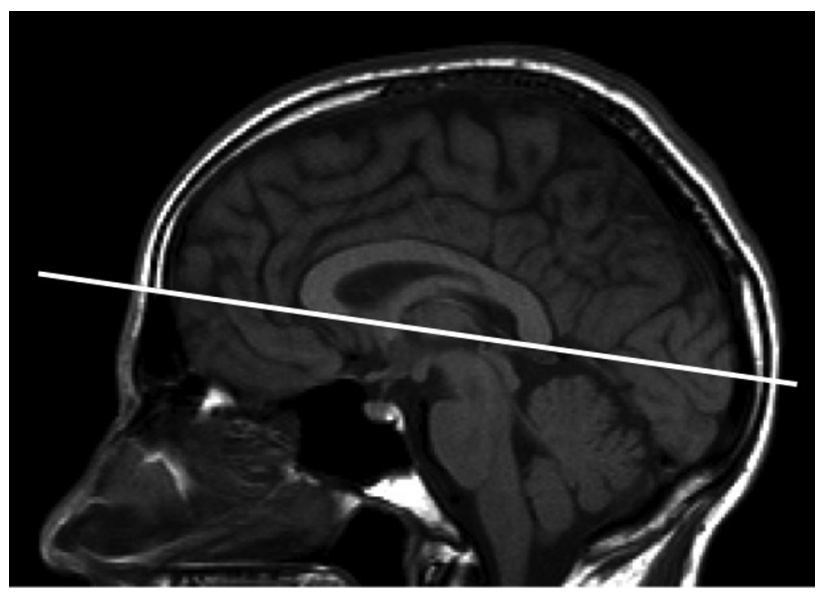

FIG 1. Orientation of axial oblique sequences. Orientation of axial oblique sequences should be along the subcallosal line as indicated by the solid line. Axial sections should be $\leq 3 \mathrm{~mm}$ with no gap.

\section{Table 2: Standardized brain MRI protocol (diagnosis and routine follow-up of MS)}

\begin{tabular}{|c|c|}
\hline Parameters & Description \\
\hline Field strength & Scans should be of good quality, with adequate SNR and resolution (in-sections, pixel resolution of $\leq 1 \times 1 \mathrm{~mm}$ ) \\
\hline Scan prescription & Use the subcallosal plane to prescribe or reformat axial oblique sections (Fig 1) \\
\hline Coverage & Whole-brain coverage \\
\hline Section thickness and gap & $\leq 3 \mathrm{~mm}$, No gap (for 2D acquisition or 3D reconstruction) \\
\hline \multirow[t]{7}{*}{ Core sequences } & Anatomic 3D inversion recovery-prepared $\mathrm{Tl}$ gradient echo (eg, 1.0- to 1.5-mm thickness) \\
\hline & Gadolinium single dose, $0.1 \mathrm{mmol} / \mathrm{kg}$ given for 30 seconds $^{\mathrm{a}}$ \\
\hline & 3D sagittal T2WI FLAIR ${ }^{b}$ (eg, 1.0- to 1.5-mm thickness) \\
\hline & $3 \mathrm{D} \mathrm{T2WI}{ }^{\mathrm{b}}$ (eg, 1.0- to 1.5-mm thickness) \\
\hline & 2D axial DWI ( $\leq 5-\mathrm{mm}$ sections, no gap) \\
\hline & 3D FLASH (non-IR prep) postgadolinium ${ }^{b}$ (eg, 1.0- to 1.5-mm thickness) \\
\hline & $\begin{array}{l}\text { 3D series would be typically reconstructed to } 3-\mathrm{mm} \text { thickness for display and subsequent comparison for } \\
\text { lesion counts }\end{array}$ \\
\hline \multirow[t]{3}{*}{ Optional sequences } & Axial proton attenuation \\
\hline & Pre- or postgadolinium axial Tl spin-echo (for chronic black holes) \\
\hline & SWI for identification of central vein within T2 lesions \\
\hline
\end{tabular}

Note:-IR indicates inversion recovery.

${ }^{a}$ Minimum 5-minute delay before obtaining postgadolinium T1. The 3D sagittal FLAIR may be acquired immediately after contrast injection before the 3D FLASH series

b If unable to perform a 3D acquisition, then perform 2D axial and sagittal FLAIR, axial fast spin-echo proton attenuation/T2, and axial post-gadolinium TIWI spin-echo at $\leq 3$-mm section thickness. 
Field strength

Scan prescription

Coverage

Sequences

Section thickness and gap
Scans should be of good quality, with adequate SNR and resolution (in-section pixel resolution of $\leq 1 \times 1 \mathrm{~mm}$ ) Use the subcallosal plane to prescribe or reformat axial oblique sections (Fig 1)

Whole-brain coverage

3D sagittal T2WI FLAIR ${ }^{\mathrm{a}}$

2D axial DWI (5-mm-thick, no gap)

$\leq 3 \mathrm{~mm}$, No gap (for 2D acquisition or 3D reconstruction)

${ }^{a}$ If unable to perform a 3D acquisition, then perform $2 \mathrm{D}$ axial FLAIR at $\leq 3$-mm section thickness.

isotropic voxels. ${ }^{7}$ The data can be reformatted to achieve 3-mm axial and/or sagittal sections for clinical readout. If a 2D acquisition is used, the section thickness should be $\leq 3 \mathrm{~mm}$ and there should be no gap between sections. 3D FLAIR may be equivalent or superior to T2-weighted imaging for posterior fossa lesions. It is recommended that a $3 \mathrm{D}$ T2 or a 3-mm axial fast/turbo spinecho proton attenuation/T2 sequence be acquired for posterior fossa lesion detection as a backup.

The brain MR imaging sequences include a sagittal FLAIR (the $3 \mathrm{D}$ acquisition is usually acquired in the sagittal plane) for the detection of MS lesions in the corpus callosum. Although these lesions can be identified on axial images, the sagittal plane provides greater ease of visualization of these, as well as juxtacortical lesions (ie, white matter lesions touching the cortex) and the oval perivenular configuration of lesions.

A thin-section ( $\leq 1.5$-mm thickness) 3D inversion recoveryprepared, T1-weighted, spoiled gradient-echo sequence is useful for volumetric analysis, which is likely to play an important role in the future. The 3D inversion recovery sequence should be acquired before contrast. This sequence also enables confirmation of juxtacortical and infratentorial lesions. Chronic T1-weighted "black hole" monitoring as a marker of severe axonal injury has only been validated on $2 \mathrm{D}$ spin-echo sequences. Because nearly all hyperintense lesions apparent on T2-weighted sequences are hypointense on $3 \mathrm{D}$ inversion recovery $\mathrm{T} 1$-weighted scans, the specificity of T1 black holes is lost and clinical interpretation requires caution. $^{8}$

The protocol includes a postgadolinium contrast 3D FLASH (non-inversion recovery prep) or axial T1-weighted spin-echo images. Although 3D gradient echo-based T1-weighted imaging could be used as a replacement for T1 spin-echo for identifying postgadolinium contrast enhancement, whether such sequences are less sensitive ${ }^{9}$ remains an open question.

Axial DWI $(5 \mathrm{~mm})$ is recommended for detecting non-MS pathology, including acute ischemia/infarction and the earlier detection of PML (see below).

The 3D or 2D FLAIR and/or T2-weighted images can be acquired during the minimum 5-minute delay that is required before the postcontrast T1-weighted image. Phased array coils may significantly speed up acquisition time.

Proton attenuation and precontrast 2D spin-echo T1weighted imaging are considered optional. Subtle lesions can be confirmed on proton attenuation imaging, though the sensitivity of 3D FLAIR may obviate this confirmation. ${ }^{10}$

Gadolinium contrast detects the breakdown of the bloodbrain barrier that occurs with new lesion development and reactivation of old lesions. The average duration of enhancement for individual brain lesions is 3 weeks, ${ }^{11}$ with most enhancing for
2-6 weeks. Rarely, MS lesions in the brain show persistent enhancement for $>3$ months with single-dose gadolinium. A standard dose of gadolinium $(0.1 \mathrm{mmol} / \mathrm{kg})$ given for 30 seconds and a minimum 5-minute delay before acquiring the postcontrast T1weighted imaging are recommended. "Triple dose" and longer delays of up to 15 minutes for the postcontrast T1-weighted imaging may detect more lesions but are not necessary for routine clinical practice. Reports of nephrogenic systemic fibrosis/nephrogenic fibrosing dermopathy in patients with preexisting significant renal impairment have resulted in many centers requiring a recent laboratory assessment of renal function, such as an estimated glomerular filtration rate. Macrocyclic chelates have been recommended by several radiologic societies to minimize this risk. $^{12,13}$

Most newly enhancing lesions will leave residual T2 hyperintensity after the enhancement resolves. ${ }^{14}$ Detecting new or enlarging T2 lesions compared with a previous study would also indicate new inflammatory activity even in the absence of gadolinium enhancement. However, to reliably detect new lesions, a standardized MR imaging protocol with similar orientation and other parameters is important. Gadolinium can also be helpful for ruling out alternative diagnoses such as tumors (persistent enhancement) or leptomeningeal disease such as neurosarcoidosis ${ }^{15,16}$ or infection.

Protocol 2: PML Surveillance Brain MR Imaging. PML is a devastating complication that is rarely seen with some disease-modifying therapies (Table 3). The risk is increased in patients with detectable John Cunningham virus serum antibodies. MR imaging detection of PML in the presymptomatic phase improves outcome and survival. ${ }^{17}$ An abbreviated PML surveillance protocol includes 3D (or 2D) FLAIR and DWI sequences. ${ }^{18}$ Postcontrast T1 adds little diagnostic value to PML surveillance because $<50 \%$ of early PML lesions show contrast enhancement. ${ }^{19}$ Typical PML lesion appearance includes subcortical lesions (48\% occur in the frontal lobes) that are hyperintense on T2/FLAIR and hypointense on T1-weighted imaging, with ill-defined borders toward the white matter and sharp borders toward the gray matter and high signal intensity on DWI (absent in about $40 \%$ of patients with presymptomatic PML). Lesions can involve the deep gray matter (thalamus and dentate nuclei). Contrast enhancement can be patchy, nodular, or speckled. ${ }^{18,19}$

Protocol 3: Spinal Cord MR Imaging. As a minimum, coverage should include the cervical cord (Table 4 ) because clinically silent MS lesions are more common and better visualized there. It may not be necessary to examine the thoracic cord routinely unless there are clinical symptoms and/or signs at that level. Two sequences are recommended for the detection of subtle lesions, including a sagittal T2-weighted and a proton attenuation, STIR, or 


\begin{tabular}{|c|c|}
\hline Parameter & Description \\
\hline Field strength & $\begin{array}{l}\text { Scans should be of good quality, with adequate SNR and resolution (in-section pixel resolution of } \leq 1 \times 1 \mathrm{~mm} \text { ) } \\
\text { Closed magnets (large bore for patients with claustrophobia) preferred }\end{array}$ \\
\hline Coverage & Cervical cord coverage $\mathrm{e}^{\mathrm{a}}$ \\
\hline Core sequences & Sagittal T2 \\
\hline & $\begin{array}{l}\text { Sagittal proton attenuation, STIR, or PST7-IR } \\
\text { Axial T2 through lesions }\end{array}$ \\
\hline Section thickness and gap & Sagittal: $\leq 3 \mathrm{~mm}$, no gap \\
\hline & Axial: $5 \mathrm{~mm}$, no gap \\
\hline Optional sequences & $\begin{array}{l}\text { Axial T2 through complete cervical cord } \\
\text { Gadolinium }{ }^{\mathrm{b}} \text { and postgadolinium sagittal } \mathrm{T} 1 \\
\text { Sagittal } T 7\end{array}$ \\
\hline
\end{tabular}

Table 5: Clinical guidelines for brain and spinal cord MRI in MS

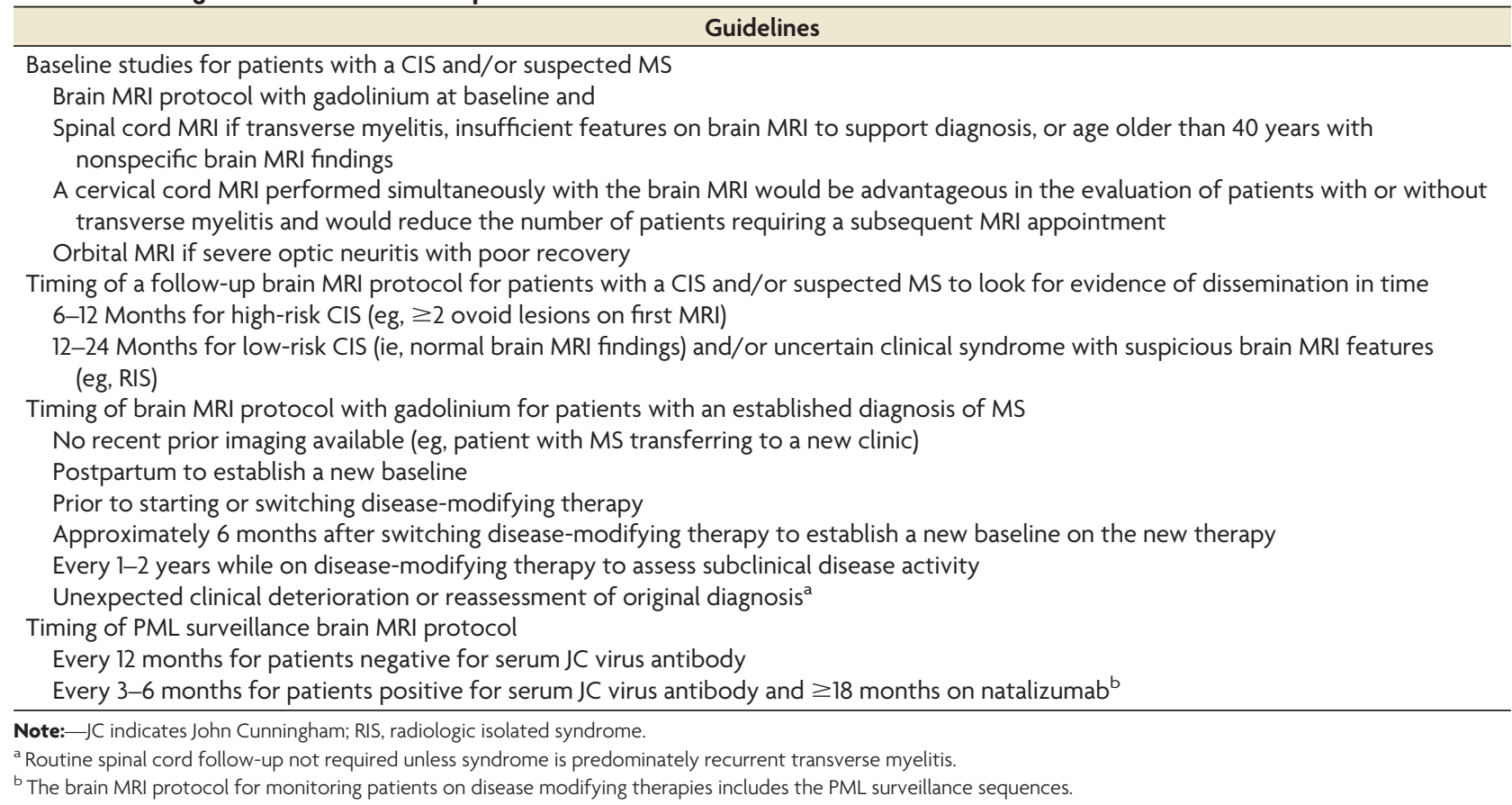

T1-weighted inversion recovery sequence with phase-sensitive reconstruction (section thickness, $\leq 3 \mathrm{~mm}$ ). ${ }^{20}$ Axial T2 or T2* and postcontrast axial $\mathrm{T} 1$ through the lesions is recommended (section thickness of $5 \mathrm{~mm}$, no gap). A sagittal T1 is of limited value for characterizing intramedullary disease. When spinal cord imaging is performed at the same time as brain imaging with gadolinium, no additional contrast is required.

Protocol 4: Orbit MR Imaging. Imaging of the orbit may be clinically indicated to confirm optic neuritis and rule out compressive lesions. Unusual enhancement patterns of the optic nerve and/or sheath might suggest an alternate diagnosis such as sarcoidosis or neuromyelitis optica. ${ }^{21}$ The recommended sequences include a coronal STIR or fat-suppressed T2 and a postgadolinium fat-suppressed $\mathrm{T} 1$ with a section thickness of $\leq 2 \mathrm{~mm}$, with coverage through the optic chiasm.

\section{Clinical Guidelines: Diagnostic Imaging for Suspected MS}

While MR imaging is not absolutely required for the clinical diagnosis of MS (Table 5), it provides important information. ${ }^{22} \mathrm{~A}$ brain MR imaging with and without gadolinium is recommended for patients suspected of having MS or with an established diagnosis of MS who are new to a clinical practice and do not have recent imaging available for review. A cervical cord MR imaging at the same time would be advantageous in the diagnostic evaluation of patients with or without transverse myelitis and would reduce the number of patients requiring a subsequent MR imaging appointment. Patients suspected of having MS include those with a CIS of optic neuritis, partial transverse myelitis, or brain stem syndromes. Patients with CIS with a brain MR imaging with $\geq 2$ characteristic lesions ( $\geq 3 \mathrm{~mm}$ in diameter) have a high risk for MS. ${ }^{23}$ One-third of patients with CIS (not treated with corticosteroids) will have asymptomatic gadolinium-enhancing lesions and will meet the $2010 \mathrm{McDonald}$ diagnostic criteria for definite MS. ${ }^{24}$ Detection of new T2 or gadolinium-enhancing lesions on a follow-up brain MR imaging can be sufficient evidence to fulfill dissemination in time and/or space criteria (Table 1). The recommended timing of the follow-up brain MR imaging is 6-12 months. The proportion of patients with high-risk CIS (younger 


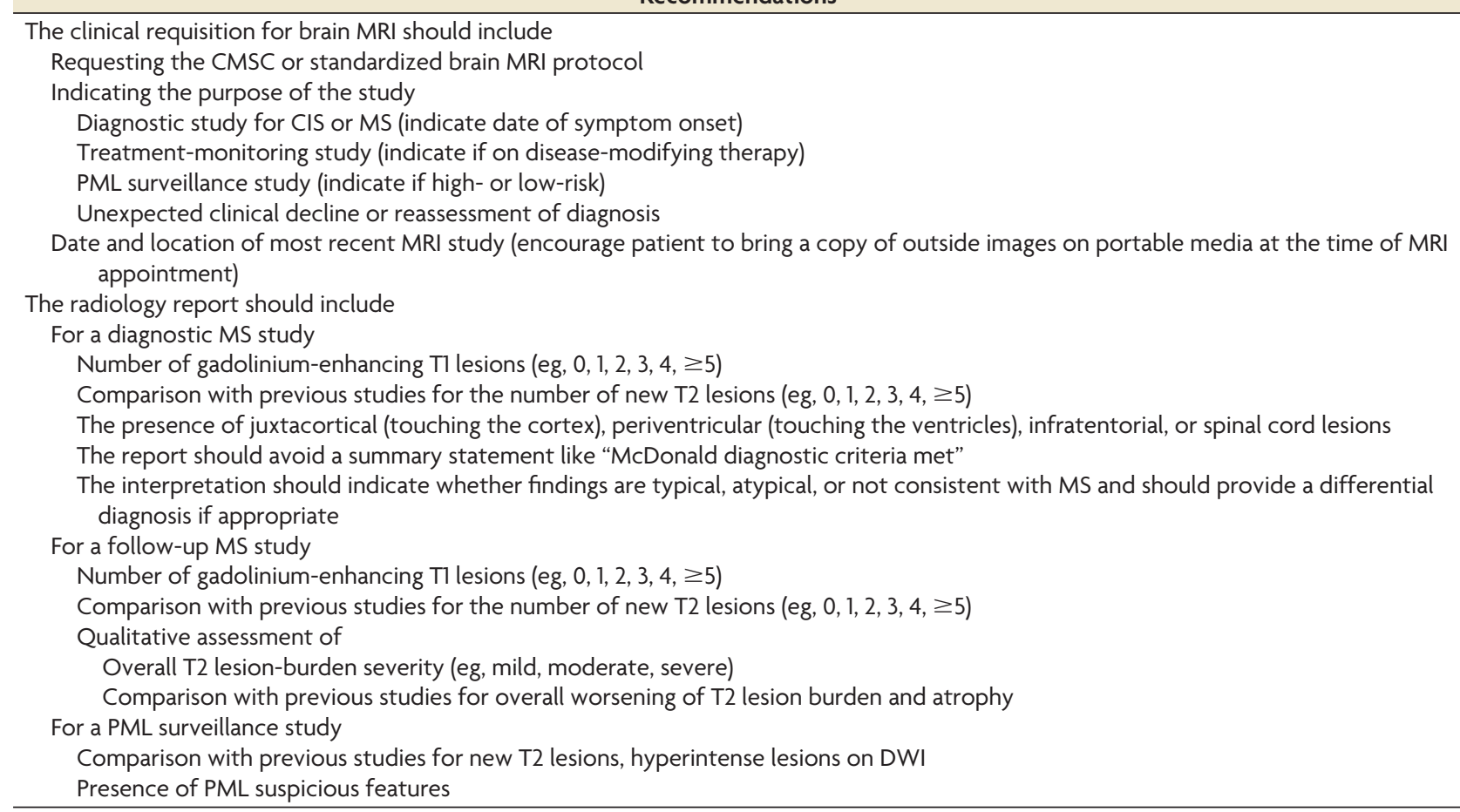

than 50 years of age) who develop new lesions by 3, 6, and 12 months is $22 \%, 51 \%$, and $74 \%$, respectively. ${ }^{25}$ By $12-18$ months, most patients with high-risk CIS will meet the diagnostic criteria for MS by developing new MR imaging lesions and/or new clinical symptoms. ${ }^{26}$ Additional MRIs can be performed according to clinical judgment. Some experts recommend an annual brain MR imaging in patients with CIS with normal brain MR imaging findings for 1-2 years or at the time of development of new symptoms consistent with demyelination. ${ }^{23,27,28}$

Patients suspected of having MS also include those with milder or atypical symptoms or incidental abnormalities on MR imaging that are strongly suspicious for MS (radiologic isolated syndrome). ${ }^{29}$ The presence of a spinal cord lesion on MR imaging may be helpful in estimating the risk of conversion to definite multiple sclerosis. ${ }^{30}$ A 5-year prospective study of 451 patients with radiologic isolated syndrome demonstrated that the risk of conversion to MS was $34 \% .{ }^{31}$ In addition to other paraclinical tests such as evoked potentials and CSF analysis, a follow-up MR imaging may support the diagnosis of MS by demonstrating new lesion development in patients suspected of having MS.

In patients with equivocal brain MR imaging findings (ie, not meeting dissemination in space criteria), the detection of lesions on spinal cord imaging can provide additional evidence for disease dissemination in space. Spinal cord lesions have greater specificity for demyelinating disease. ${ }^{31,32}$ Nonspecific white matter lesions are extremely uncommon in the spinal cord, in contrast to their frequent occurrence in the brain. ${ }^{33}$ Patients may present with a more severe demyelinating or inflammatory syndrome, including tumefactive lesions or acute complete transverse myelitis. A follow-up MR imaging would provide evidence of lesion improvement or resolution supporting an inflammatory process and/or the detection of new lesions that could aid in the differential diagnosis. ${ }^{15,34}$

Spinal cord imaging, in addition to brain imaging, is recommended if the symptoms involve the spinal cord such as partial transverse myelitis, complete transverse myelitis, or a progressive myelopathy suspicious for primary-progressive MS. Patients with progressive MS may have a chronic, diffuse, extensive abnormal spinal cord signal, though most do not. The detection of a longitudinally extensive spinal cord lesion ( $\geq 3$ segments) with central cord predominance and mass effect in a patient with acute transverse myelitis is suggestive of neuromyelitis optica. ${ }^{21}$ The longitudinally extensive involvement of these distinctive lesions is often transient.

\section{Clinical Guidelines: Follow-Up of Established MS}

The 4 common scenarios for requesting a brain MR imaging for patients with an established diagnosis of MS are the following: new baseline evaluation (previous MR imaging unavailable, unacceptable quality, or a long interval since the last MR imaging), routine follow-up for clinically silent disease activity while monitoring treatment response, PML surveillance, and unexpected clinical deterioration or re-evaluation of the diagnosis of MS (Table 6). A baseline spinal cord MR imaging may also be useful, depending on the clinical symptoms.

MR imaging is recommended before the initiation or modification of disease-modifying therapy and approximately 6 months after a treatment switch to allow sufficient time for the new therapy to reach its therapeutic potential. Determining ongoing radiologic stability is based on the presence or absence of new lesions (T2 or contrast-enhancing T1) relative to a posttreatment MR imaging. ${ }^{35}$ 
MR imaging is the most sensitive tool currently available for monitoring inflammatory disease activity in MS. Clinical assessments far underestimate disease activity and burden compared with MR imaging. A follow-up MR imaging is useful for patients on a disease-modifying therapy to determine the response of subclinical disease activity to treatment. Continued or worsening of MR imaging disease activity while on a disease-modifying therapy may prompt a change in therapy. There is evolving evidence that ongoing MR imaging activity can be indicative of a suboptimal therapeutic response. ${ }^{36-41}$

Many new lesions may be clinically silent, particularly when they occur outside the more functionally eloquent regions of the CNS (spinal cord, optic nerve). However, as more lesions accumulate in the CNS, studies demonstrate a clear relationship between the severity of cognitive dysfunction and lesion burden, even in patients with good mobility. CNS damage also occurs in brain tissue that appears normal on standard conventional imaging (normal appearing brain tissue). This slow, evolving damage can be monitored by nonconventional advanced MR imaging techniques that are mainly restricted to the research realm. New T2 or gadolinium-enhancing lesions are associated with progressive changes in normal appearing brain tissue and global brain atrophy. ${ }^{42}$

The frequency of periodic MR imaging to assess subclinical disease activity will vary depending on the patient's clinical course and other clinical features. For relapsing forms of MS, a follow-up MR imaging should be considered annually for at least the first 2 or 3 years after starting therapy or switching disease-modifying therapy. More frequent surveillance may be indicated in clinically aggressive cases or unusual patterns of MR imaging lesions (eg, tumefactive MS). Clinical judgment and experience may be critical in these settings. While guidelines on a tolerable threshold for new lesion activity that warrants a change in therapy have been proposed, ${ }^{43,44}$ individual factors will impact the clinician's decision on the frequency of MR imaging monitoring. The frequency of MR imaging may be higher during the early years for patients with CIS and early relapsing-remitting MS, particularly when patients are on treatment. Fewer MR imaging scans are required in later stages of the disease (secondaryprogressive MS) or in primary-progressive MS in which MR imaging activity is low and no effective treatments are as of yet available. ${ }^{45}$ Nonetheless, multiple new T2 or contrast-enhancing lesions on surveillance scans in conjunction with the clinical picture, even in primary-progressive $\mathrm{MS},{ }^{45}$ should alert the clinician to re-evaluate treatment strategies.

The PML surveillance recommendations by using a shorter protocol depend on John Cunningham virus serum antibody status, length of exposure to natalizumab, and the use of prior immunosuppressive therapy. ${ }^{46}$ Higher risk patients (positive for John Cunningham virus serum antibodies) with $>18$ months of natalizumab exposure should have the PML surveillance MR imaging protocol performed every 3-6 months. ${ }^{18}$ Any clinical change suspicious for PML should prompt an urgent MR imaging.

Indications for an unplanned follow-up brain MR imaging in patients with an established diagnosis of MS include the re-assessment of the original diagnosis or to clarify the cause of clinical deterioration that is not otherwise evident by clinical assessment (eg, stroke or tumor).
A spinal cord MR imaging may be indicated when there is significant clinical worsening with few changes on brain MR imaging or to rule out an alternative cause for progressive myelopathy, such as cervical spondylosis or a tumor. Routine follow-up with spinal cord imaging may be useful in rare cases of recurrent transverse myelitis to assess response to therapy or new disease activity.

\section{Recommendations for Communication and Storage}

The requisition should ask for standardized brain and/or spinal cord imaging and indicate the clinical question being addressed and include relevant clinical history (eg, CIS localization and symptom duration), current MS disease-modifying therapy, recent high-dose corticosteroids, and date and place of any previous MR imaging (Table 6).

The radiology report should use standardized terminology and include a description of salient findings. These include new T2 or contrast-enhancing T1 lesion numbers and the presence/ absence of juxtacortical, periventricular, infratentorial, and spinal cord lesions. The report should avoid a summary statement like "McDonald diagnostic criteria met," because this requires clinical details that may not be available at the time of the radiologic review. The interpretation should indicate whether the findings are typical, atypical, or not consistent with MS, and the radiologist should provide a differential diagnosis, if appropriate. In patients with definite MS, a qualitative assessment of brain volume (atrophy) and overall T2 lesion burden and a comparison with previous studies for new lesion activity and atrophy are useful (Table 6). MR imaging studies should be stored in a standard readable format (DICOM), retained permanently, and available. Patients are encouraged to keep copies of their own studies and have these available if a follow-up MR imaging is performed at a different imaging center.

\section{CONCLUSIONS}

The goal of the original guidelines was to provide clinicians with a standardized MR imaging protocol that would be suitable for the initial diagnosis and monitoring of changes with time. The protocol was designed to provide the optimum amount of information that could be acquired within a routine clinical MR imaging setting. The current revision incorporates feedback from centers using the previous protocol and input from radiologists with expertise in MS. 3D FLAIR and 3D T2-weighted imaging are already being introduced into clinical practice. These techniques can provide potentially higher quality data (higher resolution, seamless reformatting) and should be comparable with the core $2 \mathrm{D}$ approaches. The methodologies for quantification of advanced imaging techniques, such as magnetization transfer imaging, MR spectroscopy, diffusion tensor imaging, and myelin water fraction imaging, require further standardization before their routine clinical use can be recommended. Methods sensitive to gray matter pathology (double inversion recovery and phase-sensitive T1 inversion recovery sequences) are being developed but are still currently investigational. An imaging challenge for the next decade is the continued translation of research methodologies into useful and reliable clinical tools for lesion identification, quantification of T2 lesion burden, and brain and spinal cord atrophy measures. 
The revised guidelines provide consensus recommendations for the use of brain and spinal cord MR imaging and the use of gadolinium in the diagnosis of patients with MS. The challenge during the past decade was in reaching a consensus on the frequency of routine imaging to monitor patients with MS. The evidence supporting this role remains incomplete. However, there is consensus that MR imaging provides useful information about subclinical inflammatory activity in the early phase of the disease. It is reasonable for physicians to take these arguments for and against routine MR imaging into consideration when they individualize patient care.

During the past 3 decades, the clinical application of MR imaging in MS and the advances in imaging quality and speed have been remarkable. We anticipate that these guidelines will require future revision as MR imaging technology and our knowledge of MS continue to improve.

\section{ACKNOWLEDGMENTS}

Lori Saslow (Great Neck, New York) assisted in manuscript preparation and editing.

The following individuals participated in workshops and/or reviewed the guidelines: Barry Arnason, Douglas Arnold, Frederik Barkhof, Guy Buckle, Peter Calabresi, Bruce Cohen, Lesley Costley, Patricia Coyle, Virginia Devonshire, Burton Drayer, George Ebers, Massimo Filippi, Joseph Frank, Douglas Graeb, Douglas Goodin, June Halper, Colleen Harris, Stanley Hashimoto, Robert Herndon, George Hutton, Douglas Jeffrey, Ciaran Keogh, Thomas Leist, Geert Lycklama à Nijeholt, Henry McFarland, David Miller, Mary Lou Myles, Paul O’Connor, Joel Oger, Donald Paty, Daniel Pelletier, Michael Phillips, George Rice, John Richert, Jay Rosenberg, Richard Rudick, James Scott, Randall Shapiro, Nancy Sicotte, James Simsarian, Karen Smith, Jay Tsuruda, Brian Weinshenker, Ernest Willoughby, Jerry Wolinsky.

Disclosures: Anthony Traboulsee-RELATED: Support for Travel to Meetings for the Study or Other Purposes: CMSC; UNRELATED: Consultancy: Chugai Pharmaceuticals, Medlmmune, Teva Innovation, Novartis, Hoffman-La Roche, Sanofi-Genzyme, EMD Serono; Grants/Grants Pending: Chugai Pharmaceuticals, * Hoffman-La Roche,* Sanofi-Genzyme*; Payment for Lectures (including service on Speakers Bureaus): Sanofi-Genzyme. Jack H. Simon—RELATED: Support for Travel to Meetings for the Study or Other Purposes: Travel costs were covered for discussion of guidelines by CMSC; UNRELATED: Consultancy: Biogen Idec, Comments: Consultant, Advisory Board, ad hoc. Lael Stone-RELATED: Consulting Fee or Honorarium: CMSC, Comments: $\$ 500$ honorarium for participation in a meeting held in the fall of 2015; Support for Travel to Meetings for the Study or Other Purposes: CMSC, Comments: travel to Newark airport and lodging in hotel for 1 night, with meals paid directly to the hotel not to me. Elizabeth Fisher-RELATED: Consulting Fee or Honorarium: CMSC, Comments: received an honorarium for participating in the CMSC MRI guidelines-development meeting; Support for Travel to Meetings for the Study or Other Purposes: CMSC, Comments: received travel support to participate in the CMSC MRI guidelines-development meeting; UNRELATED: Consultancy: Biogen, Genzyme, Novartis, Comments: received payments for consultant work; Employment: Biogen (employed as of January 5, 2015). David E. Jones—RELATED: Consulting Fee or Honorarium: CMSC; Support for Travel to Meetings for the Study or Other Purposes: CMSC; UNRELATED: Consultancy: Genzyme, Biogen Idec, Novartis, Comments: Although most of the money went to University of Virginia for these activities, I have received personal compensation for some consulting activities with these 3 companies in the past 3 years; Grants/Grants Pending: National Multiple Sclerosis Society, ${ }^{\star}$ Biogen Idec, ${ }^{*}$ Comments: Site Principal Investigator for clinical trial with vitamin D; will be site Principal Investigator for Natalizumab as an Efficacy Switch in Participants with Relapsing Multiple Sclerosis After Failure on Other Therapies (ESCALATE); Payment for Lectures (including service on Speakers Bureaus): Program of All-inclusive Care for the Elderly, Comments: 1 lectured in 2014 (\$500); Travel) Accommodations/Meeting Expenses Unrelated to Activities Listed: Multiple Sclerosis Association of America. Jiwon Oh-RELATED: Grant: Multiple Sclerosis Society of Canada*; UNRELATED: EMD Serono, Biogen Idec, Roche, Genzyme; Grants/ Grants Pending: Biogen Idec, ${ }^{*}$ Genzyme*; Payment for Lectures (including service on Speakers Bureaus): Teva, EMD Serono, Genzyme, Biogen Idec; Travel/Accommodations/Meeting Expenses Unrelated to Activities Listed: Genzyme, EMD Serono. David S. Reich—UNRELATED: Payment for Development of Educational Presentations: University of Pennsylvania (grand rounds). Nancy Richert-UNRELATED: Employment: Biogen; Stock/Stock Options: As a Biogen employee, I received restricted stock options; Travel/Accommodations/Meeting Expenses Unrelated to Activities Listed: Biogen pays my travel and accommodations for attending national meetings (eg, American Academy of Neurology, American Society of Neuroradiology, European Committee for Treatment and Research in Multiple Sclerosis). Kottil Rammohan-UNRELATED: Consultancy: Novartis, Genentech, Genzyme, Biogen Idec, EMD Serono, Teva Neurosciences; Grants/Grants Pending: Novartis, ${ }^{*}$ Genentech, * Genzyme, ${ }^{\star}$ EMD Serono, ${ }^{\star}$ Biogen Idec, ${ }^{\star}$ Teva Neurosciences*; Payment for Development of Educational Presentations: Mallinckrodt; Travel/Accommodations/Meeting Expenses Unrelated to Activities Listed: CMSC. Omar Khan-UNRELATED: Consultancy: Teva, Biogen Idec, Genzyme, Novartis; Grants/Grants Pending: Wayne State University. Ernst-Wilhelm Radue-UNRELATED: Board Membership: Novartis, * Biogen*; Consultancy: Novartis, Biogen, Bayer Schering, Merck Serono, Genzyme, Synthon, MorphoSys; Payment for Lectures (including service on Speakers Bureaus): Novartis, Biogen, Bayer Schering, Genzyme. Corey Ford-RELATED: Grant: Teva Neuroscience, ${ }^{*}$ Comments: research grant for glatiramer acetate clinical trials including 20+ year-long term follow-up study; Consulting Fee or Honorarium: Teva Neuroscience, Comments: In the past few years, I have consulted with Teva for scientific/medical issues related to MS disease-modifying therapies, including glatiramer acetate; Support for Travel to Meetings for the Study or Other Purposes: Teva Neuroscience, Comments: travel to meetings for presentation of data and consulting; Provision of Writing Assistance, Medicines, Equipment, or Administrative Support: Teva, Comments: Teva staff participated in drafting this article and providing data from a data base maintained in multiple clinical trials of glatiramer acetate. I made substantive contributions to the writing, editing, proofing and verification of the content; UNRELATED: Consultancy: Novartis, Biogen Idec, Comments: I participated in several consulting meetings during the past 3 years. As with Teva, these were advisory-/medical-focused. I do not participate in Speakers Bureaus and all potential conflicts of interest such as these have been reported to the University of New Mexico Health Sciences Center Conflicts of Interest Committee for review and management; Grants/Grants Pending: multiple industry sponsors, ${ }^{*}$ Comments: We perform 8-10 clinical trials each year for manufacturers of MS disease-modifying drugs. All are paid to the institution and conducted under appropriate compliance and reporting guidelines; Payment for Development of Educational Presentations: CMSC, Comments: I have received compensation for the creation of Continuing Medical Education materials for the CMSC in the field of MS clinical care. June Halper-UNRELATED: Consultancy: Biogen, Comments: Delphi study group. David Li-UNRELATED: Board Membership: Opexa Therapeutics (Data Safety Advisory Board); Consultancy: Vertex Pharmaceuticals, Comments: drug-development testing; Grants/Grants Pending: Genzyme, ${ }^{*}$ Hoffman-La Roche, ${ }^{*}$ Merck Serono, ${ }^{*}$ Nuron, ${ }^{\star}$ Perceptives, ${ }^{\star}$ Sanofi-Aventis, ${ }^{*}$ Comments: I am Director of the University of British Columbia MS/MRI Research Group, which has been contracted to provide central MRI analysis for therapeutic trials in multiple sclerosis. *Money paid to the institution.

\section{REFERENCES}

1. Polman CH, Reingold SC, Banwell B, et al. Diagnostic criteria for multiple sclerosis: 2010 revisions to the McDonald criteria. Ann Neurol 2011;69:292-302 CrossRef Medline

2. Dalton CM, Brex PA, Miszkiel KA, et al. Application of the new McDonald criteria to patients with clinically isolated syndromes suggestive of multiple sclerosis. Ann Neurol 2002;52:47-53 CrossRef Medline

3. Poser CM, Paty DW, Scheinberg L, et al. New diagnostic criteria for multiple sclerosis: guidelines for research protocols. Ann Neurol 1983;13:227-31 CrossRef Medline

4. Harris JO, Frank JA, Patronas N, et al. Serial gadolinium-enhanced magnetic resonance imaging scans in patients with early, relapsingremitting multiple sclerosis: implications for clinical trials and natural history. Ann Neurol 1991;29:548-55 CrossRef Medline

5. Simon JH, Li D, Traboulsee A, et al. Standardized MR imaging protocol for multiple sclerosis: Consortium of MS Centers consensus guidelines. AJNR Am J Neuroradiol 2006;27:455-61 Medline

6. Wattjes MP, Barkhof F. High field MRI in the diagnosis of multiple sclerosis: high field-high yield? Neuroradiology 2009;51:279-92 CrossRef Medline

7. Vrenken H, Jenkinson M, Horsfield MA, et al; MAGNIMS Study 
Group. Recommendations to improve imaging and analysis of brain lesion load and atrophy in longitudinal studies of multiple sclerosis. J Neurol 2013;260:2458-71 CrossRef Medline

8. Barkhof F, Karas GB, van Walderveen MA. T1 hypointensities and axonal loss. Neuroimaging Clin N Am 2000;10:739-52, ix Medline

9. Rand S, Maravilla KR, Schmiedl U. Lesion enhancement in radiofrequency spoiled gradient-echo imaging: theory, experimental evaluation, and clinical implications. AJNR Am J Neuroradiol 1994; 15:27-35 Medline

10. Gramsch C, Nensa F, Kastrup O, et al. Diagnostic value of 3D fluid attenuated inversion recovery sequence in multiple sclerosis. Acta Radiol 2015;56:622-27 CrossRef Medline

11. Cotton F, Weiner HL, Jolesz FA, et al. MRI contrast uptake in new lesions in relapsing-remitting MS followed at weekly intervals. Neurology 2003;60:640 - 46 CrossRef Medline

12. Nacif MS, Arai AE, Lima JAC, et al. Gadolinium-enhanced cardiovascular magnetic resonance: administered dose in relationship to United States Food and Drug Administration (FDA) guidelines. J Cardiovasc Magn Reson 2012;14:18 CrossRef Medline

13. Thomsen HS, Morcos SK, Almén T, et al; ESUR Contrast Medium Safety Committee. Nephrogenic systemic fibrosis and gadoliniumbased contrast media: updated ESUR Contrast Medium Safety Committee guidelines. Eur Radiol 2013;23:307-18 CrossRef Medline

14. Miller DH, Rudge P, Johnson G, et al. Serial gadolinium enhanced magnetic resonance imaging in multiple sclerosis. Brain 1988: 111(pt 4):927-39 CrossRef Medline

15. Traboulsee A, Li DK. Conventional MR imaging. Neuroimaging Clin N Am 2008;18:651-73, x CrossRef Medline

16. Charil A, Yousry TA, Rovaris M, et al. MRI and the diagnosis of multiple sclerosis: expanding the concept of "no better explanation." Lancet Neurol 2006;5:841-52 CrossRef Medline

17. Dong-Si T, Richman S, Wattjes MP, et al. Outcome and survival of asymptomatic PML in natalizumab-treated MS patients. Ann Clin Transl Neurol 2014;1:755-64 CrossRef Medline

18. Wattjes MP, Barkhof F. Diagnosis of natalizumab-associated progressive multifocal leukoencephalopathy using MRI. Curr Opin Neurol 2014;27:260-70 CrossRef Medline

19. Yousry TA, Pelletier D, Cadavid D, et al. Magnetic resonance imaging pattern in natalizumab-associated progressive multifocal leukoencephalopathy. Ann Neurol 2012;72:779-87 CrossRef Medline

20. Poonawalla AH, Hou P, Nelson FA, et al. Cervical spinal cord lesions in multiple sclerosis: T1-weighted inversion-recovery MR imaging with phase-sensitive reconstruction. Radiology 2008;246:258-64 CrossRef Medline

21. Wingerchuk DM, Banwell B, Bennett JL, et al. International consensus diagnostic criteria for neuromyelitis optica spectrum disorders. Neurology 2015;85:177-89 CrossRef Medline

22. McDonald WI, Compston A, Edan G, et al. Recommended diagnostic criteria for multiple sclerosis: guidelines from the International Panel on the Diagnosis of Multiple Sclerosis. Ann Neurol 2001;50: 121-27 CrossRef Medline

23. Brex PA, Ciccarelli O, O'Riordan JI, et al. A longitudinal study of abnormalities on MRI and disability from multiple sclerosis. NEngl J Med 2002;346:158-64 CrossRef Medline

24. Kang H, Metz LM, Traboulsee AL, et al; Minocycline in CIS Study Group. Application and a proposed modification of the $2010 \mathrm{Mc}-$ Donald criteria for the diagnosis of multiple sclerosis in a Canadian cohort of patients with clinically isolated syndromes. Mult Scler 2014;20:458-63 CrossRef Medline

25. Edan G, Kappos L, Montalbán X, et al; BENEFIT Study Group. Longterm impact of interferon beta-1b in patients with CIS: 8-year follow-up of BENEFIT. J Neurol Neurosurg Psychiatry 2014;85:1183-89 CrossRef Medline

26. Beck RW, Chandler DL, Cole SR, et al. Interferon beta-1a for early multiple sclerosis: CHAMPS trial subgroup analyses. Ann Neurol 2002;51:481-90 CrossRef Medline
27. The Optic Neuritis Study Group. Multiple sclerosis risk after optic neuritis: final optic neuritis treatment trial follow-up. Arch Neurol 2008;65:727-32 doi: CrossRef Medline

28. Fisniku LK, Brex PA, Altmann DR, et al. Disability and T2 MRI lesions: a 20-year follow-up of patients with relapse onset of multiple sclerosis. Brain 2008;131(pt 3):808-17 CrossRef Medline

29. Okuda DT, Mowry EM, Beheshtian A, et al. Incidental MRI anomalies suggestive of multiple sclerosis: the radiologically isolated syndrome. Neurology 2009;72:800-05 CrossRef Medline

30. Okuda DT, Mowry EM, Cree BA, et al. Asymptomatic spinal cord lesions predict disease progression in radiologically isolated syndrome. Neurology 2011;76:686-92 CrossRef Medline

31. Okuda DT, Siva A, Kantarci O, et al. Radiologically isolated syndrome: 5-year risk for an initial clinical event. PLoS One 2014;9: e90509 CrossRef Medline

32. Sombekke MH, Wattjes MP, Balk LJ, et al. Spinal cord lesions in patients with clinically isolated syndrome: a powerful tool in diagnosis and prognosis. Neurology 2013;80:69-75 CrossRef Medline

33. Bot JC, Barkhof F, Polman CH, et al. Spinal cord abnormalities in recently diagnosed MS patients: added value of spinal MRI examination. Neurology 2004;62:226-33 CrossRef Medline

34. Miller DH, Weinshenker BG, Filippi M, et al. Differential diagnosis of suspected multiple sclerosis: a consensus approach. Mult Scler 2008;14:1157-74 CrossRef Medline

35. Simon JH, Bermel RA, Rudick RA. Simple MRI metrics contribute to optimal care of the patient with multiple sclerosis. AJNR Am J Neuroradiol. 2014;35:831-32 CrossRef Medline

36. Rudick RA, Lee JC, Simon J, et al. Significance of T2 lesions in multiple sclerosis: a 13-year longitudinal study. Ann Neurol 2006;60: 236-42 CrossRef Medline

37. Sormani MP, Bonzano L, Roccatagliata L, et al. Magnetic resonance imaging as a potential surrogate for relapses in multiple sclerosis: a meta-analytic approach. Ann Neurol 2009;65:268-75 CrossRef Medline

38. Prosperini L, Gallo V, Petsas N, et al. One-year MRI scan predicts clinical response to interferon beta in multiple sclerosis. Eur J Neurol 2009;16:1202-09 CrossRef Medline

39. Bermel RA, You X, Foulds P, et al. Predictors of long-term outcome in multiple sclerosis patients treated with interferon $\boldsymbol{\beta}$. Ann Neurol 2013;73:95-103 CrossRef Medline

40. Sormani MP, Rio J, Tintorè M, et al. Scoring treatment response in patients with relapsing multiple sclerosis. Mult Scler 2013;19: 605-12 CrossRef Medline

41. Rio J, Castilló J, Rovira A, et al. Measures in the first year of therapy predict the response to interferon beta in MS. Mult Scler 2009;15: 848-53 CrossRef Medline

42. Chard DT, Brex PA, Ciccarelli O, et al. The longitudinal relation between brain lesion load and atrophy in multiple sclerosis: a 14 year follow up study. J Neurol Neurosurg Psychiatry 2003;74:1551-54 CrossRef Medline

43. Multiple Sclerosis Therapy Consensus Group (MSTCG), Wiendl H, Toyka KV, Rieckmann P, et al. Basic and escalating immunomodulatory treatments in multiple sclerosis: current therapeutic recommendations. J Neurol 2008;255:1449-63 CrossRef Medline

44. Freedman MS, Selchen D, Arnold DL, et al; Canadian Multiple Sclerosis Working Group. Treatment optimization in MS: Canadian MS Working Group updated recommendations. Can J Neurol Sci 2013; 40:307-23 CrossRef Medline

45. Hawker K, O'Connor P, Freedman MS, et al; OLYMPUS trial group. Rituximab in patients with primary progressive multiple sclerosis: results of a randomized double-blind placebo-controlled multicenter trial. Ann Neurol 2009;66:460-71 CrossRef Medline

46. Bloomgren G, Richman S, Hotermans C, et al. Risk of natalizumabassociated progressive multifocal leukoencephalopathy. $N$ Engl J Med 2012;366:1870-80 CrossRef Medline 